\title{
IMPORTÂNCIA DA ATENÇÃO MULTIDISCIPLINAR PARA RESGATAR O PACIENTE COM HIV/AIDS APRESENTANDO BAIXA ADESÃO À TERAPIA ANTIRRETROVIRAL
}

\author{
IMPORTANCE OF MULTIDISCIPLINARY CARE TO RESCUE THE PATIENT WITH HIVIAIDS \\ PRESENTING POOR ADHERENCE TO ANTIRETROVIRAL THERAPY
}

\section{Natália Raguzzoni Cancian ${ }^{a^{*}}$, Sandra Trevisan Beck ${ }^{b^{*}}$, Gilvane Souza dos Santos ${ }^{\mathrm{c}^{*}}$, Danieli Bandeira ${ }^{\mathrm{d}^{*}}$}

anaticancian@hotmail.com, bsbeck@ig.com.br, cgilvanesm@yahoo.com.br, ddanielibandeira22@gmail.com

*Universidade Federal de Santa Maria - Santa Maria (RS), Brasil

Data de recebimento do artigo: 27/08/2014

Data de aceite do artigo: 09/03/2015

\section{RESUMO}

Introdução: No Brasil, milhares de pessoas sofrem as consequências clínicas da infecção pelo HIV, a qual é enfrentada através da distribuição gratuita de medicamentos antirretrovirais pelo Ministério da Saúde. Objetivo: Identificar, entre pacientes vivendo com HIV/AIDS em terapia antirretroviral (TARV), fatores que os levaram a não aderir ao tratamento. Material e métodos: Entre abril e novembro de 2013, foram agendados 637 pacientes para consulta nos ambulatórios de doenças infecciosas do Hospital Universitário de Santa Maria e do Centro de Testagem e Aconselhamento Casa Treze de Maio de Santa Maria, Rio Grande do Sul. Neste período, foi realizada a busca ativa de 90 pacientes não aderentes à TARV, através da ação da Residência Multiprofissional em Saúde. 39 pacientes compareceram à consulta multiprofissional, com farmacêutico, enfermeiro e nutricionista. Foi realizada escuta qualificada, seguida de orientaçóes referentes às diferentes áreas profissionais. A adesão foi avaliada pelo Cuestionario para la Evaluación de la Adhesión al Tratamiento Antiretroviral (CEAT-VIH) [Questionário para a Avaliação da Adesão ao Tratamento Antirretroviral], o estado nutricional pelo questionário Malnutrition Universal Screening Tool (MUST) [Ferramenta Universal para Rastreio da Malnutrição], além de entrevistas semiestruturadas. Resultados: Os 39 pacientes foram reconduzidos à TARV. Os fatores identificados como importantes para a má adesão foram baixa escolaridade e informação insuficiente sobre a medicação utilizada. A melhora significativa da adesão ao tratamento após a consulta multiprofissional foi comprovada através da mudança nos resultados da carga viral. Conclusão: $O$ restabelecimento do vínculo do paciente com a equipe de saúde é um fator fundamental para a adesão à TARV.

Palavras-chave: AIDS; atenção à saúde; comunicação interdisciplinar.

\section{ABSTRACT}

Introduction: In Brazil, thousands of people suffer clinical consequences of HIV infection, which is tackled by free distribution of antiretroviral drugs by the Ministry of Health. Objective: Identify among patients living with HIV/AIDS on antiretroviral therapy (ART), factors that led to nonadherence to treatment. Methods: Between April and November 2013, 637 patients were scheduled for consultation in outpatient infectious diseases, at the University Hospital of Santa Maria and at the center for counseling and Testing House Thirteen May of Santa Maria, Rio Grande do Sul. During this period was made the active search for 90 non-adherent patients on ART by the action of the multidisciplinary Residency in Health. 39 patients attended the multidisciplinary consultation with pharmacist, nurse and nutritionist. Qualified hearing followed by guidelines for the different professional areas was performed. Compliance was assessed by Cuestionario para Evaluación de la Adhesión al Tratamiento Antiretroviral (HIV-CEAT) [Assessment of Adherence to Antiretroviral Therapy Questionnaire], nutritional status by questionnaire Malnutrition Universal Screening Tool (MUST) and by semistructured interviews. Results: The 39 patients were reappointed to ART. The factors identified as important for poor adherence are poor education and 
insufficient information about the used medication. The significant improvement in treatment adherence after multidisciplinary consultation was confirmed by the change in viral load results. Conclusion: The restoration of the link with the patient's healthcare team is critical to ART adherence factor.

Keywords: AIDS; health care; interdisciplinary communication.

\section{Introdução}

Segundo o Boletim Epidemiológico HIV/AIDS de 2012, o Brasil tem 656.701 casos registrados de AIDS, ou seja, pessoas sofrendo as consequências clínicas da infecção pelo HIV. Em 2011, foram notificados 38.776 casos da doença, e a taxa de incidência de AIDS no Brasil foi de 20,2 casos por 100 mil habitantes ${ }^{1}$.

No Brasil, dentre as estratégias para combater a epidemia, destaca-se a política de distribuição universal e gratuita dos medicamentos antirretrovirais pelo Ministério da Saúde. A Terapia Antirretroviral (TARV) ofertada aos portadores do HIV que necessitam de tratamento desde 1996 reduziu a morbimortalidade, taxa de internaçóes, e melhorou a expectativa de vida dessas pessoas $^{2}$.

A atenção ambulatorial a esses pacientes tem sido dada como nas demais doenças crônicas, por causa das associaçóes terapêuticas e ao acesso que a população infectada tem aos medicamentos antirretrovirais ${ }^{3}$.

Com a distribuição gratuita de antirretrovirais, vários aspectos melhoraram, como: diminuição nas internaçôes hospitalares; redução da mortalidade e redução da transmissão vertical, entre outros ${ }^{4}$. Porém, para que os aspectos positivos sejam alcançados, e a TARV seja efetiva, é necessária uma estreita adesão ao regime prescrito, pois o uso irregular ou em doses insuficientes pode propiciar o desenvolvimento de vírus HIV resistente.

É importante ressaltar que os atuais esquemas terapêuticos são complexos e de difícil adesão, além de serem associados a reaçóes adversas e interaçóes medicamentosas. O uso do "coquetel" anti-HIV trouxe efeitos colaterais, como náuseas, vômitos, diarreia, anorexia, diabetes melito, lipodistrofia, entre outros ${ }^{3}$. Esses aspectos negativos acabam vinculados à náo adesáo da terapia medicamentosa, sendo a causa mais comum da falha do tratamento, e a principal variável na qual os serviços de saúde podem intervir para aumentar a efetividade da medicação.

Porém, a adesão à TARV vai além do uso correto do medicamento, frequência das doses e possíveis reaçóes adversas. Consiste também na falta de informação, pobreza, preconceito e uso de drogas ${ }^{6}$. A equipe de saúde, através do relato do usuário e da compreensão desses fatores relacionados à adesão, pode ajudar no entendimento do tratamento ${ }^{7}$.
A equipe multidisciplinar, composta por diferentes profissionais da saúde, entre eles enfermeiro, nutricionista e farmacêutico, veio substituir aquele cuidado focado apenas no médico, agregando mais valor ao cuidado do paciente. A consulta da nutrição, enfermagem, juntamente da farmácia, reforçam o uso correto da medicação, monitora os exames laboratoriais realizados, que melhoram a qualidade nutricional e de vida do paciente, levando a um menor índice de infecçoos secundárias e internações, melhor prognóstico e menores custos para o sistema de saúde . $^{8}$.

Para isso, é fundamental a equipe multidisciplinar somar esforços para facilitar esse processo, estimulando o autocuidado?. O paciente deve entender e aceitar a prescrição, e como trata-se de um processo dinâmico, a equipe de saúde é corresponsável pela adesão ${ }^{10}$.

O presente estudo teve como objetivo identificar, durante uma consulta multidisciplinar, quais são os fatores, medicamentosos ou náo, que levam o paciente à não adesão do tratamento, permitindo assim a implementação de estratégias que melhorem a aceitação da sua condição de vida.

\section{Materiais e métodos}

\section{Casuística}

Entre abril e novembro de 2013, foram agendados 637 pacientes para consulta nos ambulatórios de doenças infecciosas do Hospital Universitário de Santa Maria (HUSM) e do Centro de Testagem e Aconselhamento Casa Treze de Maio de Santa Maria, Rio Grande do Sul (RS). Nesse período, foi realizada a busca ativa de 90 pacientes não aderentes à TARV. A amostra foi composta por pacientes em TARV que apresentaram falha virológica, detectada através de exames laboratoriais. Dos 90 pacientes selecionados, 39 compareceram à consulta multiprofissional, cuja equipe era formada por profissional farmacêutico, nutricionista e enfermeiro, que faziam parte do programa de Residência Multiprofissional em Saúde, do Hospital Universitário de Santa Maria (RS). Houve perda de 51 indivíduos, que apesar da tentativa de contato telefônico por pelo menos cinco vezes, não foram encontrados ou náo foram receptivos ao convite da equipe. Entre eles, um foi a óbito, dois estavam acamados e dois estavam em regime privado de liberdade. 


\section{Critérios de inclusão}

Foram incluídos no estudo pacientes maiores de 18 anos, em TARV há pelo menos 24 semanas, que apresentaram falha virológica definida por não obtenção ou não manutenção de carga viral indetectável na circulação sanguínea após início de tratamento medicamentoso.

Caracteriza-se como falha virológica a detecção de carga viral acima de 400 cópias virais/mL após 24 semanas, acima de 50 cópias virais/ $\mathrm{mL}$ após 48 semanas, ou para indivíduos que atingiram supressão viral completa (vírus não detectável na corrente sanguínea), e voltaram a apresentar, na vigência de TARV, carga viral acima de 400 cópias $/ \mathrm{ml}^{11}$.

\section{Critérios de exclusão}

Foram excluídos do estudo indivíduos menores de 18 anos, gestantes (por seguirem protocolo especial de tratamento), indivíduos com dificuldade cognitiva, acamados, em privaçáo de liberdade, além dos que não concordaram em participar do estudo, ou assinar o Termo de Consentimento Livre e Esclarecido (TCLE).

\section{Metodologia}

A consulta multiprofissional foi realizada através do acolhimento do usuário e dos esclarecimentos de suas dúvidas, traçando seu perfil socioeconômico por meio de questionário semiestruturado da enfermagem. $\mathrm{O}$ aspecto nutricional foi verificado através da aplicação do questionário validado Malnutrition Universal Screening Tool (MUST) [Ferramenta Universal para Rastreio da Malnutrição], e a atenção farmacêutica foi realizada seguindo um protocolo de atendimento, orientado pela aplicação de um questionário, que possibilitou a verificação de fatores importantes relacionados à adesão do tratamento, o Cuestionario para la Evaluación de la Adhesión al Tratamiento Antirretroviral (CEAT-VIH) [Questionário para a Avaliação da Adesão ao Tratamento Antirretroviral]. Este questionário, de origem espanhola, foi validado experimentalmente para o Brasil por Remor et al. ${ }^{12}$. É um questionário autoaplicável, e possui vinte itens de caráter multidimensional, que englobam os principais fatores capazes de moldar o comportamento de adesão do paciente ao tratamento. Os principais são: administração da medicação pelo paciente; intensidade dos efeitos colaterais; avaliar se ele se sente capaz ou náo de seguir o tratamento; avaliação do paciente sobre o próprio estado de saúde; e sua relação com o médico ${ }^{12}$.
O questionário foi aplicado de forma verbal, cuidando para não ter influência sobre as respostas dadas. A cada pergunta foi estipulado um valor, e a pontuação total foi obtida pela soma de todos os itens, sendo o valor mínimo 17 pontos e o máximo 89. Quanto maior a pontuaçáo, maior o grau de adesão ao tratamento. De acordo com as análises de validação do questionário, os pacientes que obtiveram uma pontuação menor que 74 foram classificados com aderência insuficiente; os que obtiveram pontuação entre 75 a 79 apresentaram boa aderência. Quando a pontuação atingiu 80 a 89 pontos, o paciente foi considerado estritamente aderente ${ }^{12}$.

O estudo foi submetido e aprovado pelo Comitê de Ética em Pesquisa da Universidade Federal de Santa Maria, sob o CAAE: 20005613.3.0000.5346.

\section{Resultados}

Após a busca ativa dos pacientes não aderentes à TARV, pôde ser avaliado o perfil dos que compareceram à consulta multiprofissional. Ela foi composta de indivíduos com média de 41 anos (a idade mínima foi 27 e a máxima 59), predominando o sexo feminino (21 de 39 pacientes). No que se refere ao grau de instrução, a maioria dos pacientes $(56,41 \%)$ possuía escolaridade baixa, com o ensino fundamental incompleto (Tabela 1).

Tabela 1: Escolaridade dos pacientes não aderentes à terapia antirretroviral.

\begin{tabular}{lc}
\hline \multicolumn{1}{c}{ Escolaridade } & Número de pacientes \\
\hline Ensino fundamental incompleto & 22 \\
Ensino fundamental completo & 3 \\
Ensino médio incompleto & 4 \\
Ensino médio completo & 7 \\
Ensino superior incompleto & 2 \\
Ensino superior completo & 1 \\
\hline
\end{tabular}

Chama a atenção, na análise da aplicação desse questionário, o fato da não adesão ao tratamento não se relacionar com a intensidade dos efeitos colaterais, pois dos 39 pacientes, 20 deles $(51,28 \%)$ disseram ter efeitos colaterais pouco ou nada intensos. Porém, quando avaliados pelo nutricionista, 25 pacientes relataram ter efeitos colaterais relacionados ao trato gastrintestinal, como náusea e diarreia, e 10 tiveram outros sintomas, como alucinação, mostrando a importância da abordagem multidisciplinar para fornecer atenção integral ao paciente. Sintomas semelhantes foram relatados durante a entrevista com a enfermagem. 
O grau de aderência ao tratamento antirretroviral, após o CEAT-VIH, confirmou que todos os pacientes avaliados apresentaram pontuação menor que 74 , ou seja, aderência insuficiente ao tratamento, como mostra a Tabela 2 .

Tabela 2: Distribuição dos pacientes segundo o grau de aderência à terapia antirretroviral.

\begin{tabular}{lc}
\hline Grau de Aderência (pontos) & Pacientes (n) \\
\hline Estrita (80-89) & 0 \\
Boa (75-79) & 0 \\
Insuficiente (menor que 74) & 39 \\
\hline
\end{tabular}

Foi possível detectar algumas discrepâncias entre a percepção de alguns pacientes quanto ao esforço relatado para seguir o tratamento e a tomada regular da medicação. Entre os 39 pacientes avaliados, 19 relataram fazer muito esforço para seguir o tratamento, 17 consideravam fazer esforço regular e 3 pacientes não se consideravam esforçados (Tabela 3).

Tabela 3: Esforço para seguir o tratamento e ingestão correta das medicações.

\begin{tabular}{cccc}
\hline \multirow{2}{*}{ Esforço } & \multicolumn{3}{c}{ Ingestáo correta da medicaçáo } \\
\cline { 2 - 4 } & Muito/bastante & Regular & Pouco/nada \\
\hline Muito/bastante & 13 & 4 & 2 \\
Pouco/regular & 3 & 8 & 6 \\
Nada & 1 & 2 & 0 \\
Total & 17 & 14 & 8 \\
\hline
\end{tabular}

A maioria dos pacientes que se consideravam bastante esforçados para seguir o tratamento cumpriam a tomada de medicaçóes. Dos três pacientes que se consideravam nada esforçados, um deles alegou cumprir assiduamente a tomada (ingestão) das medicações, como também pode ser visto na Tabela 3 .

A relação com o médico é outro ponto importante quando se fala em adesão ao tratamento antirretroviral. Dos pacientes entrevistados, 34 deles diziam ter uma boa relação com o médico, mas apenas 15 tinham muita/bastante informação sobre a medicação utilizada. Por outro lado, dois usuários diziam ter uma relação regular/ruim com o médico, mas um deles tinha muita/ bastante informação (Tabela 4).

Após a consulta dos pacientes, pôde-se averiguar a importância do atendimento multiprofissional, através do retorno à TARV, confirmado pelos resultados laboratoriais. Após a intervenção, o resultado geral foi positivo, pois 22 pacientes obtiveram redução ou não detecção da carga viral, mostrando a conscientização do usuário em tratamento (Tabela 5).
Tabela 4: Nível de informação sobre a terapia antirretroviral relacionado ao atendimento médico entre os pacientes não aderentes.

\begin{tabular}{lccc}
\hline \multirow{2}{*}{ Relaçáo com médico } & \multicolumn{3}{c}{ Informaçáo sobre medicaçáo } \\
\cline { 2 - 4 } & Muito/bastante & Regular & Pouco/nada \\
\hline Boa & 15 & 5 & 14 \\
Pode melhorar & 1 & 1 & 1 \\
Regular/ruim & 1 & 1 & 0 \\
Total (n) & 17 & 7 & 15 \\
\hline
\end{tabular}

Tabela 5: Alteração dos resultados das cargas virais verificado após consulta multidisciplinar.

\begin{tabular}{cc}
\hline Pacientes $(\mathbf{n})$ & Avaliaçáo da carga viral \\
\hline 6 & Passaram a valores Indetectáveis \\
16 & Reduziram os níveis \\
2 & Não tinham resultados anteriores \\
14 & Não reduziram os níveis \\
1 & Não tinha resultado posterior \\
\hline
\end{tabular}

\section{Discussão}

Vários estudos têm sido desenvolvidos nos últimos anos, enfatizando a importância da aderência ao tratamento antirretroviral, buscando diferentes fatores que possam ser relevantes no momento em que se inicia o tratamento dos indivíduos com AIDS. Recentemente, Resende et al. ${ }^{13}$ estimaram o grau de adesão ao tratamento e os fatores que poderiam intervir nele, realizando a pesquisa em Unidade de Dispensação de Medicamentos (UDM) e Centro de Testagem e Aconselhamento (CTA) de Alfenas, Minas Gerais, utilizando o mesmo questionário (CEAT- VIH) em pacientes usuários de medicamentos antirretrovirais, destacando maior participação do sexo masculino. No presente estudo, houve predominância de mulheres em busca do atendimento multiprofissional oferecido. Esse perfil, diferente do encontrado na literatura, pode ser explicado pela inclusão de apenas pacientes não aderentes no estudo, e não todos os pacientes recebendo TARV, mostrando que a mulher é mais sensível aos apelos realizados para cuidados com a saúde.

Contudo, para que se tenha uma real compreensão da importância do acompanhamento clínico e da administração correta na medicação, é necessário um grau satisfatório de instrução que permita assimilar a importância dos cuidados propostos pela equipe de saúde. O baixo nível de escolaridade encontrado entre os indivíduos atendidos pela equipe multiprofissional mostra que este é um dos prováveis fatores que levaram os pacientes a abandonarem inicialmente a TARV. Estudos anteriores mostram que a adesão insuficiente ao tratamento antirretroviral está relacionada com a 
pouca escolaridade da amostra estudada, tendo em vista a complexidade do tratamento e da compreensão da doença. Um estudo de coorte realizado em pacientes de Porto Alegre (RS) após seis meses de início de terapia antirretroviral, cujo objetivo foi avaliar os fatores predisponentes de falha virológica, identificou que a não adesão e a baixa escolaridade mantiveram-se associadas à falha virológica ${ }^{14,15,16,17}$. Também constataram que a uma boa escolaridade contribui para a melhor adesão.

Outro fator importante para a não adesão são os efeitos colaterais provocados pelos medicamentos usados, como neuropatia, lipodistrofia, pancreatite, hepatotoxicidade, diabetes, dislipidemia e osteoporose. Os sintomas, geralmente leves, como náuseas, vômitos, cefaleia e dor abdominal, tendem a desaparecer após as primeiras semanas de tratamento, justificando o estudo que relata que, apesar de importantes, os efeitos colaterais são insuficientes para explicar a não adesão ao tratamento ${ }^{18}$, e que mesmo apresentando algum mal-estar, a maioria faz uso regular dos antirretrovirais ${ }^{17}$. Porém, mesmo de forma intermitente, esses efeitos, quando presentes, contribuem para uma pior qualidade de vida do indivíduo com HIV/AIDS ${ }^{6}$. Como nem sempre os sintomas adversos são vinculados pelo paciente ao medicamento ingerido, o atendimento multiprofissional (que trabalha com múltiplas identidades de interaçáo e qualidade de vida do usuário $^{19}$ ) pode detectar intercorrências, que passariam despercebidas, caso o atendimento seja centrado em um único profissional.

A pouca compreensão da prescrição, aliada à falta de informações sobre os riscos da náo adesão ao tratamento, estáo entre os muitos fatores que dificultam a adesão à terapia antirretroviral ${ }^{6}$. Não é raro que muitos pacientes tenham vontade de seguir o tratamento e digam-se aderentes, mas quando o questionamento por parte do profissional de saúde é aprofundado, eles mostram não usar corretamente a medicação, talvez porque não tenham sido sensibilizados o suficiente pela equipe que os acompanha quanto às informaçóes necessárias para o tratamento ${ }^{20}$. Uma vez que as pessoas tendem a não tomar os medicamentos corretamente se não sabem como fazê-lo, fornecer informaçóes sobre os medicamentos mostra-se uma atividade fundamental.

Cardoso e Arruda ${ }^{10}$ compararam dois grupos de pacientes: os aderentes e os não aderentes. Esse estudo constatou que, para o grupo dos aderentes, a relação com o médico é ótima e ele é visto como um amigo, resultando numa boa interação entre os dois. Para os não aderentes não há essa relação, e eles inclusive evitam o contato com o médico, mesmo havendo, por parte deste, um interesse na aproximação. Tal resultado é diferente do encontrado neste estudo, pois a maioria tem um bom relacionamento, e mesmo assim é considerada não aderente. A boa relação com o médico não se mostrou suficiente para se ter um bom nível de informação sobre o tratamento, como mencionado anteriormente na Tabela 4.

A pouca compreensão das informaçóes sobre o tratamento e o não entendimento da posologia prescrita são fatores que levam à não adesão. Uma comunicação inadequada está sendo vinculada à utilização errada do esquema antirretroviral. A literatura sugere que seja entregue material informativo por escrito e tabelas com os horários de ingestáo dos medicamentos, que reforçariam as informaçóes repassadas verbalmente ${ }^{21,22,23}$. É necessário que essa informação seja fornecida de maneira clara e adequada, para facilitar sua retenção, o que implicaria em questôes mais profundas do que simplesmente divulgar conhecimento. Deve-se abranger estratégias que contribuam para a tomada de decisóes, mudança de atitudes e comportamento que, sem dúvidas, serão positivamente influentes para aprofundar o vínculo entre o paciente e a equipe de saúde ${ }^{18}$. Um enfoque multiprofissional pode proporcionar isto ${ }^{24}$.

Apesar de ter ocorrido perda de 51 pacientes durante a busca ativa, 39 usuários de TARV foram reconduzidos ao tratamento correto, mostrando vínculo com a equipe de saúde e o retorno às consultas agendadas, tanto pelo médico, quanto pela equipe multiprofissional. Esses pacientes, apresentando menor carga viral devido à adesáo do tratamento antirretroviral, provavelmente terâo menor número de internaçóes hospitalares e de gastos com o tratamento de agravos, e consequentemente, melhora na qualidade de $\mathrm{vida}^{25}$.

\section{Conclusão}

O papel da equipe multiprofissional que presta assistência às pessoas vivendo com HIV/AIDS tem de ser pautado pelo vínculo com o paciente, baseado na confiança estabelecida entre equipe e usuário. A orientação sobre a doença, medicamentos e efeitos colaterais deve ser conduta de qualquer profissional responsável por esse cuidado, principalmente dos nutricionistas, enfermeiros e farmacêuticos.

Estratégias como grupos de adesão ou consultas individuais mais interativas são necessárias para que a equipe possa, além de falar, ouvir as queixas e dúvidas dos pacientes. Todo o tratamento deve ser revisado, valendo-se da escuta qualificada para identificar as possíveis falhas, e assim melhorá-las. O profissional deve ser corresponsável pelo tratamento, mas também deve empoderar o paciente, sugerindo que ele desenvolva o questionamento e a criticidade, além de buscar informaçóes sobre sua saúde. O respeito pelas diferenças deve prevalecer, pois com isso facilitamos o processo de criação do vínculo entre médico e paciente, a adesão ao tratamento, além de estabelecermos o princípio da equidade, tão sonhado no Sistema Único de Saúde. 


\section{Referências}

1. Ministério da Saúde. Secretaria de Vigilância em Saúde. Programa Nacional de DST e Aids. Boletim Epidemiológico - HIV/AIDS. Dez 2012;1[aproximadamente 60 p.]. Disponível em: http://www.aids.gov.br/sites/default/files/ anexos/publicacao/2012/52654/boletim_2012_final_1_ pdf_21822.pdf. [acesso em 14 set 2015].

2. Padoin SMM, Paula CC, Zuge SS, Primeira MR, Santos EEP, Tolentino LC. Fatores associados à não adesão ao tratamento antirretroviral em adultos acima de 50 anos que têm HIV/AIDS. DST - Jornal Brasileiro de Doenças Sexualmente Transmissíveis. 2011;23(4):194-7.

3. Coppini LZ, Ferrini MT. Síndrome da Imunodeficiência Adquirida (AIDS). In: Guia de Nutrição: Nutrição Clínica no Adulto. Editora Manole Ltda. Barueri. 2002;235-47.

4. Ministério da Saúde. Boletim Epidemiológico - AIDS, ano XVI. mar 2002;1:[aproximadamente 70 p.]. Disponível em: http://bvsms.saude.gov.br/bvs/periodicos/bol_marco_2002.pdf. [acesso em 14 set 2015].

5. Nemes MIB, Carvalho HB, Souza MFM. Antiretroviral therapy adherence in Brazil. Aids. 2004;(18):15-20.

6. Ministério da Saúde. Recomendaçôes para a terapia anti-retroviral em adultos e adolescentes infectados pelo HIV. Coordenação Nacional de DST e AIDS; 2004. Dísponível em: http://www.giv.org.br/publicacoes/consenso_2004.pdf. [acesso em 14 set 2015].

7. Russell CK, Bunting SM, Graney M, Hartig MT, Kisner P, Brown B. Factors that influence the medication decision making of persons with HIV/AIDS: A taxonomic exploration. Journal of the Association of Nurses in AIDS Care. 2003;14(4):46-60.

8. Kotler DP. Nutritional alterations associated with HIV infection. Journal of Acquired Immune Deficiency Syndromes. 2000;(25):81-7.

9. Martins S, Martins T. Adesão ao tratamento antirretroviral: vivências de escolares. Texto Contexto Enfermagem. 2011;20(1):111-8.

10. Cardoso GP, Arruda A. As representaçóes sociais da soropositividade e sua relação com a observância terapêutica. Ciência e Saúde Coletiva. 2004;10(1):151-62.

11. Gallant JE. Approach to the treatment-experienced patient. Infectious Disease Clinics of North America. 2007;21(1):85-102.

12. Remor E, Milner-Moskovics J, Preussler G. Adaptação brasileira do "Cuestionario para la Evaluación de la Adhesión al Tratamiento Antiretroviral”. Revista de Saúde Pública. 2007;41(5):685-94.

13. Resende RC, Podestá MHMC, de Souza WA, Barroso TDO, Da Costa VB, Gomes OM, Ferreira EB. Adesão ao tratamento antirretroviral de pacientes vivendo com HIV/
AIDS atendidos pelo Sistema Único de Saúde. Revista da Universidade Vale do Rio Verde. 2012;10(2):186-201.

14. Tuboi SH, Harrison LH, Sprinz E, Albernaz RK, Schechter M. Predictors of virologic failure in HIV-1-infected patients starting highly active antiretroviral therapy in Porto Alegre, Brazil. Journal of Acquired Immune Deficiency Syndromes. 2005;(40):324-8.

15. Nemes MIB. Aderência ao tratamento por anti-retrovirais em serviços públicos de saúde no Estado de São Paulo. 2000: Ministério da Saúde,Brasília.

16. Blatt CR, Citadin CB, Souza FGD, Mello RSD, Galato D. Avaliação da adesão aos anti-retrovirais em um município no Sul do Brasil. Rev Soc Bras Med Trop. 2009;42(2):131-6.

17. Almeida ELD, Araújo GBDS, Santos VA, Bustorff LACV, Pereira AVDL, Dias MD. Adesão dos portadores do HIV/ AIDS ao tratamento: fatores intervenientes. Revista Mineira de Enfermagem. 2011;15(2):208-16.

18. Melchior R, Nemes MIB, Alencar TMD, Buchalla CM. Desafios da adesão ao tratamento de pessoas vivendo com HIV/Aids no Brasil. Revista de Saúde Pública. 2007;41(2):87-93.

19. Martinelli ML. O Trabalho do assistente social em contextos hospitalares: desafios cotidianos. Revista Serviço Social e Sociedade. Set 2011;(107):479-505.

20. Silva ALCND, Waidman MAP. Adesão e não-adesão à terapia anti-retroviral: as duas faces de uma mesma. Revista Brasileira de Enfermagem. Abr 2009;62(2):213-20.

21. Fincham JE, editor. Advancing prescription medicine compliance: new paradigms, new practices. New York: Pharmaceutical Products Press. 1995;3(2).

22. Tailor SA, Foisy MM, Tseng A, Beardsall A, Ostrop N, Khaliq Y, Hughes C. The role of the pharmacist caring for people living with HIV/AIDS: a Canadian position paper. Canadian Journal of Hospital Pharmacy. 2000;53(2):92-103.

23. Isacson D, Bingefors K. Attitudes towards drugs - a survey in the general populations. Pharmacy world and science. 2002;(24):104-10.

24. Ceccato MDGB, Acurcio FA, de Fátima Bonolo P, Rocha GM, Guimarães MD. Compreensão de informaçóes relativas ao tratamento anti-retroviral entre indivíduos infectados pelo HIV. Cad. Saúde Pública. 2004;20(5):1388-97.

25. Ministério da Saúde. Secretaria de Vigilância em Saúde. Programa Nacional de DST e Aids. Manual de adesão ao tratamento para pessoas vivendo com HIV e aids. 2008. Disponível em: http://www.aids.gov.br/ sites/default/files/Manual_de_adesao_web.pdf. [acesso em 15 set 2015 ]. 